\title{
ALGORITHMISCHE UNTERSUCHUNGEN VON BMAP/G/1 BEDIENUNGSMODELLEN MIT PAUSENZEITEN
}

\author{
Helmut Schellhaas, Technische Hochschule Darmstadt
}

Bedienungsmodelle mit Pausenzeiten haben im Hinblick auf wichtige Anwendungen (etwa) in der Informatik (Rechnerverkehrstheorie) reges Interesse gefunden. Wir betrachten Einbedienermodelle mit zwei verschiedenen Pausenpolitiken, nämlich der T-Politik und der k-Politik. In beiden Modellen legt der Bediener Pausen ein, sobald das System leer wird. Während bei der T-Politik die Bedienung wieder beginnt, sobald bei einem Pausenende mindestens ein Kunde im System ist, beginnt bei der k-Politik die Bedienung wieder, sobald mindestens k Kunden im System sind. Ankunftsprozeß ist der von Lucantoni (1991) eingeführte Markovsche Ankunftsprozeß mit Gruppen (BMAP), der eine ganze Reihe bekannter Ankunftsprozesse als Spezialfälle umfaßt (Poisson-Prozeß, PhasenErneuerungsprozeß, Markov-modulierter Poisson-Prozeß, Neuts' versatiler Markovscher Punktprozeß).

Ziel der Untersuchungen sind Darstellungen der stationären Verteilung der Kundenzahl (und gewisser Hilfsvariabler) in numerisch brauchbarer Form. Dazu werden zunächst geeignete eingebettete Markov-Ketten mi den modernen auf M.F. Neuts zurückgehenden matrix-analytischen Methoden untersucht. Es wir $\downarrow$ gezeigt, daß bekannte algorithmische Techniken für Matrix-M/G/1- Modelle Verwendung finden können. Darauf aufbauend werden mit Hilfe der Theorie der semi-regenerativen Prozesse algorithmische Lösungen für die zeitstationäre Verteilung der Kundenzahl entwickelt. Die Berechnung dieser Verteilung kann rekursiv erfolgen basierend auf der stationären Verteilung der eingebetteten Markov-Kette, für die algorithmische Lösungen vorliegen. Schließlich werden die Bedienungsmodelle mit einer Bewertungsstruktur verknüpft und die Kostenrate bestimmt.

Eine ausführliche Darstellung findet sich in:

H. Schellhaas: Single server queues with a batch Markovian arrival process and server vacations. Preprint-Nr. 1566, Fachbereich Mathematik, Technische Hochschule Darmstadt, 1993. Erscheint in: Operations Research Spektrum. 